\title{
Optical Measurements at the Combustor Exit of the HIFiRE 2 Ground Test Engine
}

\author{
Michael S. Brown* \\ Air Force Research Laboratory, Propulsion Directorate, WPAFB, OH, 45433 \\ Gregory C. Herring, ${ }^{\dagger}$ Karen Cabell, ${ }^{*}$ and Neal Hass ${ }^{\S}$ \\ NASA Langley Research Center, Hampton, VA 23861 \\ Todd F. Barhorst, ${ }^{* *}$ and Mark Gruber ${ }^{\dagger \dagger}$ \\ Air Force Research Laboratory, Propulsion Directorate, WPAFB, OH, 45433
}

\begin{abstract}
The development of optical techniques capable of measuring in-stream flow properties of air breathing hypersonic engines is a goal of the Aerospace Propulsion Division at AFRL. Of particular interest are techniques such as tunable diode laser absorption spectroscopy that can be implemented in both ground and flight test efforts. We recently executed a measurement campaign at the exit of the combustor of the HIFiRE 2 ground test engine during Phase II operation of the engine. Data was collected in anticipation of similar data sets to be collected during the flight experiment. The ground test optical data provides a means to evaluate signal processing algorithms particularly those associated with limited line of sight tomography. Equally important, this in-stream data was collected to compliment data acquired with surface-mounted instrumentation and the accompanying flowpath modeling efforts - both CFD and lower order modeling. Here we discuss the specifics of hardware and data collection along with a coarse-grained look at the acquired data and our approach to processing and analyzing it.
\end{abstract}

\section{Acronyms}

AFRL - Air Force Research Laboratory

AHSTF - Arc-heated Scramjet Test Facility

ART - Algebraic reconstruction tomography

DFB - Distributed feedback diode

DSTO - Australian Defense Science and Technology Organization

HDCR - HIFiRE Direct-Connect Rig

HIFiRE - Hypersonic International Flight Research Experimentation

LOS - Lines of sight (optical)

NIR - Near infrared

TDLAS - Tunable Diode Laser Absorption Spectroscopy

\section{Introduction}

HIFiRE is a joint hypersonic flight test program established to collect basic research data using several flight vehicles with a variety of objectives and vehicle platform characteristics through a team effort involving AFRL, DSTO and NASA. The HIFiRE Flight 2 experiment seeks to investigate dual-mode to full scramjet mode transition and performance of a hydrocarbon-fueled engine operating between Mach 6 and $8 .^{1}$ The secondary research

\footnotetext{
${ }^{*}$ Research Physicist, AFRL/RZAS, AIAA Associate Fellow.

${ }^{\dagger}$ Research Physicist, Advanced Sensing and Optical Measurement Branch

* Aerospace Engineer, Hypersonic Airbreathing Propulsion Branch.

$\S$ Aerospace Engineer, Hypersonic Airbreathing Propulsion Branch, AIAA Senior Member.

${ }^{* *}$ Research Engineer, AFRL/RZAS, AIAA Member.

† Prinicipal Aerospace Engineer, AFRL/RZAS, AIAA Associate Fellow.
} 
objectives include providing for a test bed of diode laser-based instrumentation and the acquisition of core flow combustion products (water) up through Mach 8 scramjet flight conditions. Opto-mechanical hardware aimed at meeting these objectives has been designed for integration into the flight payload. An optically-accessible flange will be placed at the exit of the combustor just upstream of the bifurcated exhaust nozzle. The flange provides for 8 optical lines of sight -5 vertical and 3 horizontal. These lines of sight (LOS) enable water-based absorption measurements. Two flight electronics packages fiber-coupled to the flange contain NIR diode lasers and photodectors that will yield absorption spectra of water via tunable diode laser absorption spectroscopy (TDLAS). ${ }^{2}$ In direct support of the flight experiment development, an engine built to the same flow path lines and scale (HIFiRE Direct-Connect Rig - HDCR) has been tested in direct-connect fashion in the AHSTF at NASA Langley. Phase I test results and companion CFD results have recently been reported. ${ }^{3,4,5}$ The ground-test engine provides an opportunity for making absorption measurements at the same axial flowpath location as the intended flight instrument. A research-grade TDLAS instrument was recently integrated onto the HDCR for data collection during the Phase II tests. The goal of the absorption measurements is multi-fold: 1) to explore data processing algorithms that can in turn be applied to data captured during flight (tomography in particular), 2) to better assess anticipated optical signal levels and their associated uncertainties recorded during flight, and 3) provide data feedback for the CFD and lower order modeling efforts associated with the ground-test activities. The TDLAS instrument was previously demonstrated on the DCR II engine at ATK-GASL for a sequence of runs at a simulated flight conditions near Mach 7. Its hardware and software architecture was derived from in-house work at AFRL. ${ }^{6,7,8,9}$

\section{Hardware}

Both the flight instrument and the ground-test instrument take advantage of distributed feedback (DFB) diode lasers developed for telecommunication applications. The diodes have central emission wavelengths near $1.4 \mu \mathrm{m}$ where numerous rovibrational water transitions are found. These transitions have been well characterized by other research groups and agreement over line strengths is quite good with uncertainties less than $2 \%$ for several of the

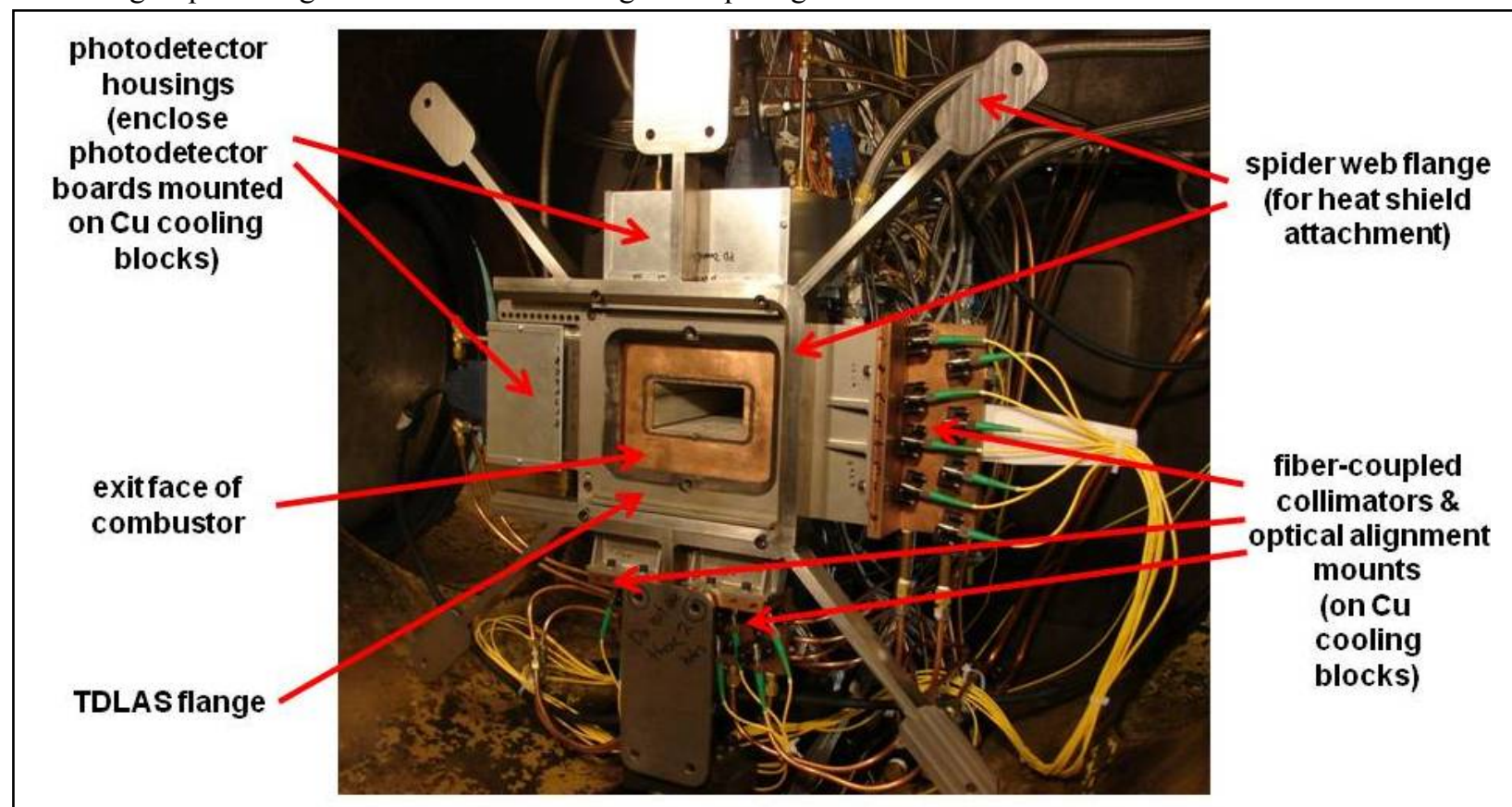

Figure 1. TDLAS flange and associated hardware attached to combustor exit of HIFiRE 2 ground test engine.

accessible lines. Both instruments use multiple transitions to extract temperature, pressure and water mole fraction. The spectra are acquired in direct scan mode implementing InGaAs photodetectors coupled to transimpedance amplifiers.

The differences between the flight and ground test hardware systems largely have to do with the constraints imposed on flight experiment instrumentation. These constraints lead to trade-off strategies as the desire for as much in-flight data acquisition as possible is balanced with upper limits on hardware power consumption, weight, size, 
and telemetry bandwidth. Additionally, the flight hardware has to be constructed such that it survives the anticipated flight environment with respect to vibration, mean acceleration, and payload temperature excursions. The testing environment and hardware constraints on the ground-test system are significantly less imposing. Consequently, this system was designed to capture more data (higher collection rate, more lines of sight, and more absorption features) than the flight system over a sample of test conditions mimicking those anticipated in flight. The strategy here being to develop data reduction tools and understanding on the more comprehensive ground test data set that can then be successfully applied to the expected flight test data set.

The flight electronics boxes make use of a single DFB that is current tuned at $50 \mathrm{kHz}$ across four water transitions with central optical frequencies of $7160.187,7160.235,7160.812$, and $7161.410 \mathrm{~cm}^{-1}$. The resulting spectra are averaged in real time and reported to the flight telemetry system at $170 \mathrm{~Hz}$. The ground-based instrument employs 3 diodes that are serially tuned at a rate of $1 \mathrm{kHz}$ and the raw signals are preserved without averaging. The use of 3 diodes enables the capture of an additional half dozen lines (including 7179.752, 7181.156, and 7185.597 $\mathrm{cm}^{-1}$ ) not captured by the flight instrument permitting trade-off comparisons of the accuracy of derived quantities such as temperature with tuning range/speed. The 3-laser tuning scheme also enables the capture of non-resonant transmission signals that reflect local optical opacity and density fluctuations.

The flight instrument uses fiber optics to both couple diode laser light to the optical flange and return collected light to the electronics boxes. The design emphasizes robustness at the expense of ease of alignment. The ground test instrument uses fiber optics for light delivery directly across the flowpath and onto the photodetector/amplifier pairs providing alignment flexibility (refer to Fig. 1). The fiber-delivery collimator heads are captured in smallfootprint high-resolution adjustment mounts. The fiber pitches and mounts for the horizontal LOS are easily seen on the right hand side of Fig.1. The corresponding photodetectors are enclosed in the Al box on the opposite side of the flow path opening. Each photodetector is mounted on a custom-designed electronics board that includes the transimpedance amplifiers.

The flight flange employs 8 LOS arranged in a rectilinear grid with each vertical LOS parallel to the others and perpendicular to the horizontal LOS. For risk reduction, the 8 LOS are evenly split between two supporting electronics boxes that house identical laser/photodetector/amplifier/DAQ setups. The flange used in the ground test work is not constrained by the flight requirements for size, strength, etc. and employs 14 LOS across the combustor exhaust opening ( 8 vertical and 6 horizontal) - some are arranged parallel to a side of the combustor exit and some are intentionally angled. This scheme permits acquisition of data that can be used to better explore tomography algorithms to generate temperature and water density spatial maps. Two additional horizontal lines of sight traverse the exit plane of the combustor just outside of the core flow enabling a measure of the environmental conditions outside of the core flow. The ground test measurements were made at the exit plane of the combustor in a windowless manner. Hence, for thermal protection, the fiber launches and corresponding photodetector boards were placed a few inches away from the flowpath opening in the transverse direction as seen in Fig. 1. The beams crossed the exit-plane opening about one-quarter inch downstream of this plane.

The InGaAs photodectors used in the ground tests are sensitive between 0.8 and $1.8 \mu \mathrm{m}$. Consequently, they are sensitive not only to the diode laser light but also to radiation from the combustion exhaust gases over this spectral range. In work done in the scramjet models at WPAFB, we have found it necessary to block some of this radiation which is largely due to hot water and carbon dioxide in order to maintain signals within the dynamic range of the detectors. This can be successfully accomplished using 0.6-mm thick slabs cut from Si wafers. Use of commercially available narrow-band filters was found to be less satisfactory due to poor transmission performance over the narrow spectral region of the diode output. The Si filters were implemented for the data collection runs on the HDCR by placing them directly in front of the photodetector boards.

For testing, the HDCR was mounted inside a continuously evacuated test cabin and directly coupled through a facility nozzle to the arc heater. For multiple reasons including convenience and hardware survivability, only the fibers, optical mounts, and photodetector boards were placed inside the test cabin. The fibers and power and data cables were passed through the forward test cabin bulkhead to an instrument cart that could be operated remotely from the facility control room. The transmission/absorption data for all $16 \mathrm{LOS}$, a reference intensity photodiode, and a solid etalon were all recorded using custom-written software operating on National Instruments DAQ hardware. The etalon transmission traces were used to transform the time-dependent diode laser sweeps into optical frequency sweeps. The diode lasers were repeatedly swept at $1 \mathrm{kHz}$ and the photodetector/amplifier pairs were sampled at $1 \mathrm{MHz}$. The software routine also permitted the recording of a synchronization pulse to better enable proper time alignment between the collected optical data and the collected facility data consisting of wall pressures and temperatures along with air and fuel rates, operating condition of the arc, etc. The gain setting for each transimpedance amplifier was independently adjusted through the software routine, and at times adjusted between runs of differing simulated flight conditions. 
In the absence of a true exhaust section, the flow exiting the engine behaves in an under expanded mode and recirculation flow sets up in the test cabin. To help protect instrumentation from recirculation hot gases, a $\mathrm{Cu}$ heat shield of nearly the same diameter as the test cabin was attached to the optical mounting flange on the pads noted in Fig. 1. The shield prevented most of the engine effluent from migrating upstream of the exit plane.

For each engine run, optical data collection began before the arc was lit and was terminated after fueling of the engine ceased. In this manner the pre and post run "health" of the optical system associated with each run could be easily evaluated. Similarly the background water contribution to the absorption measurements could be assessed for each run. Additionally, performing optical data collection over the entire facility procedure enables better synchronization with the facility data set for occurrences of compromised synch signal detection.

\section{Signal processing}

A sample of a raw acquired optical signal is shown in Fig. 2. (The DAQ software recorded each raw signal trace without averaging making it possible to look at changes on the millisecond time or scale or longer time scales by averaging during post processing.) The three diode lasers are independently and serially swept in time (timemultiplexing) and appear as the three voltage "ramps." For a brief period of time, at the onset of each ramp all diodes are in the off state. Each diode is swept in time across an optical frequency range that encompasses local

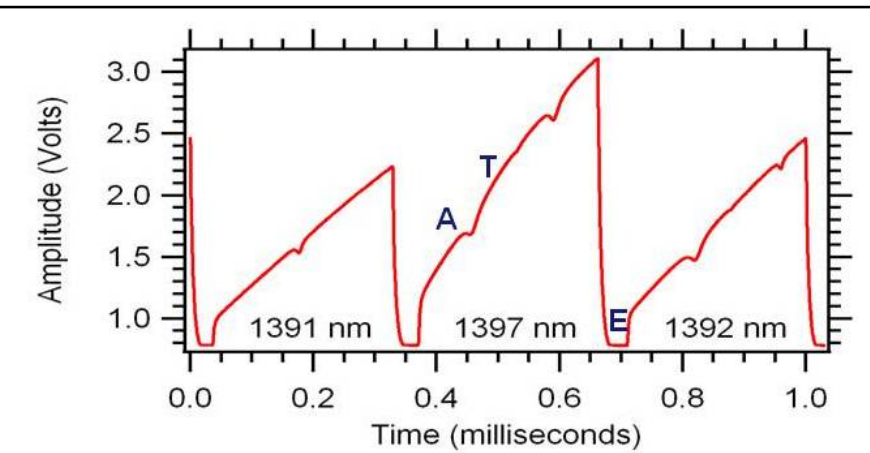

Figure 2. Example of single raw optical signal indicating three types of signals present: $A$ - resonant absorption, $T$ - nonresonant transmission, and $\mathrm{E}$ - emission. regions of absorption due to water transitions as well as regions of no absorption. This signal collection scheme permits separation of three distinct signal types that carry complimentary information. In Fig. 2 we denote examples of three such regions by the letters A, T, and $\mathrm{E}$. When the diodes are off, the photodetectors record only emission radiating from the hot combustion gases (E). ${ }^{10}$ Near water transition frequencies, (A), absorption signals are collected - five transitions are visible in the figure. Between these features non-resonant transmission signals are recorded $(\mathrm{T})$. In data processing, the emission signals (E) are simply averaged together over the short time period of their isolation from the other two types of signals. The emission can then be studied independently as well as be removed from the absorption and transmission signals via simple scalar subtraction. Each absorption feature is processed separately with a local baseline removal executed using low-order polynomial fits to the baseline regions on both sides of each transition. The net result is a spectral line that is then numerically integrated resulting in a line absorbance value. The set of absorbance values for a given 1-ms sweep are then used to derive a temperature and water concentration value via a Boltzmann plot analysis assuming validity of Beer's law. The non-resonant transmission signals are processed simply by isolating segments between the absorption features and executing an un-weighted average to generate a scalar representative of the transmission for that particular millisecond in time. Other methods to analyze the transmission signal are also available by looking at the deviations about the mean over the full segment. During signal processing the signal from each LOS is processed independently.

Since the individual diode laser beam LOS pass across regions both inside and outside of the flow, the absorption signals include contributions from the water present in the flow and the water present in the ambient test cabin environment. The signal from the two regions are simply additive for the data collected. The absorption signal due to the test cabin environment was found from the two horizontal LOS that did not pass through the flow field. This ambient absorbance was subtracted from the total signal for each line of sight prior to extraction of temperature and water concentration. As noted below, the correction for ambient water is quite small under the engine test conditions of this study.

With large data sets, it is not possible to humanly examine all spectra recorded for anomalies or artificial outliers due to electronic noise transients or beam obscuration. Identification of such was made by examining how closely grouped to a line the absorbance values for the individual spectral features appeared in the Boltzmann extraction of 
temperature. Spectra for which exceptionally poor groupings occurred (indicating the transient presence of electric noise for example) are removed from any data averaging.

Tomographic reconstruction of the temperature and water concentration fields is achieved using numerical techniques specific to algebraic reconstruction tomography (ART). These numerical approaches are distinct from that widely used in the medical community for MRI and CT imaging. Medical imaging employs many thousands of LOS along sets of parallel paths acquired at numerous azimuthal angles with respect to the sample volume. Such data sets are well analyzed by the family of techniques known as filtered back projection which rely heavily on FFT routines. These techniques perform rather poorly for sparse data sets with LOS that are not necessarily parallel to their neighbors. The imaging community has therefore pursued alternative numerical approaches such as algebraic reconstruction tomography (ART). We have adopted one of these by following the procedures noted by Sidky ${ }^{11}$ and co-workers. In brief, the approach makes a guess at the true "image" and then refines the guess iteratively. From the iterated image, a set of values for the measured quantity along the measurement LOS is numerically constructed and compared with the measured values (of temperature for example). The image is iterated until the difference between the calculated values (of temperature) along the sampled LOS and the measured values is minimized. In this sense, the algorithm is attempting to interpolate the parameter of interest in the regions between the LOS. Without refinement such algorithms can introduce high frequency "noise" in the parameter value in the interpolated regions. Similar to the approach discussed by Sidky we refine the basic iteration steps in two ways. First we ensure that the parameter value associated with each pixel remains positive and does not "race off" toward large unphysical values. Second we examine the spatial gradient of the parameter value at each pixel to ensure that large gradients are not developing. If one is found, then the most recent iterated change to that pixel is moderated.

In our preliminary look at the data collected, we have found the tomography routine to be robust and convergent to a solution independent of the initial guess. It does however "treat" the vertical LOS differently than the horizontal LOS. At times the solution image of temperature or water concentration is characterized by vertical LOS values slightly higher than those measured and horizontal LOS slightly lower. We are currently consulting experts in the field of tomography to find additional refinements to our process to mitigate this.

The operators of the AHSTF know from

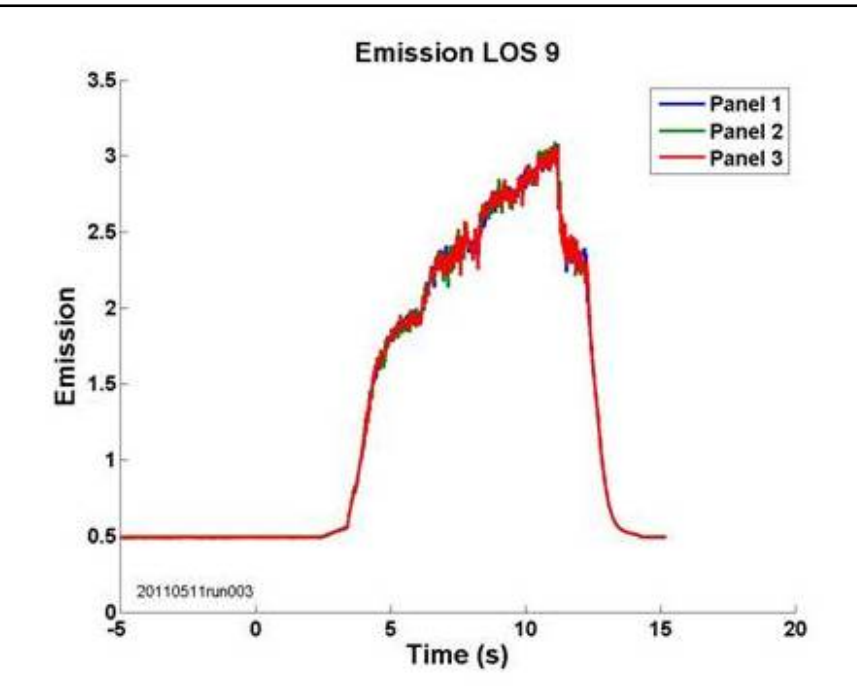

Figure 3. Example of emission signal for horizontal line of sight. Traces are shown for each of the 3 diodeoff time periods during a single sweep as a function of time.

reduced. previous experience that the arc sheds fine particulates of copper oxides during operation. Our first pass in examining the collected data was partly designed to look for instances in which a signal channel might reveal temporary particulate obscuration or a transient emission from an incandescing particle. Very few such instances were found. Some data sets were however compromised by emission signal levels that pushed the upper limit of the operational range of the photodetector/amplifier pairs. A doubling of the thickness of the Si filters used alleviated much of this and analyzable data was captured for nearly all simulated flight Mach numbers and fuel schedule.

Based on signal-to-noise ratios and the known uncertainties of the underlying spectroscopic constants used, the extracted temperature and water concentration values have uncertainties on the order of $\sim 5 \%$. The tomographic maps of temperature and water density have uncertainties presently estimated at $\sim 10 \%$. It remains a part of the ongoing research to determine if this uncertainty can be

\section{Processed data samples}

The contribution of background water to the absorption signal due just to the arc heater appears to be negligible with observed water concentration levels at or near the detection limit of the TDLAS system. Examination of the absorption signals acquired along the two horizontal paths outside of the flow reveal that the test cabin water 
concentration is low and does not significantly change during a run. This indicates that there is no "migration" of water from the core flow into the surrounding environment in the vicinity of the exit plane. Likewise, recirculation within the test cabin does not change the ambient water concentration much at all in the neighborhood of the optical measurements. Some heating is observed - the extracted temperature before a run is typically a little below $300 \mathrm{~K}$ and during the run abruptly rises to values of $450-550 \mathrm{~K}$ once the combustor is lit. When fueling ceases the temperature promptly drops to very near pre-run levels. The behavior suggests that the temperature rise is driven by radiative heating from the exiting core flow.

In Fig.3 we show an example of an emission signal recorded along one of the horizontal lines of sight for a complete engine run at a flight enthalpy of Mach 6.5. The combustor was lit near 3.5 seconds on the timeline. The rounded step appearance of the signal post ignition reflects the stepped fueling which began at a global equivalence ratio of 0.7 and was discretely increased 3 times to a total ratio of 1.1. The emission steps are coincident in time with the fueling changes. Their roundedness reflects the relative heat-soaked state of the engine. As the wall temperature rises with each fueling change, the loss of heat to the walls from the flow drops. The reduced heat loss is manifested in the increased emission. The observed emission is a signature of the radiation load on the combustor. As scramjet engines are scaled to larger sizes, this radiation load will become increasingly important and is now the subject of at least one independent study. We have recently initiated an information exchange with researchers at the University of Michigan in an attempt to quantify the observed emission. ${ }^{12}$

Examination of samples of the non-resonant transmission signals reveals two phenomena. First, slow long-time drift in the mean signal levels are observed. This is most likely due to thermal growth of elements in the optical hardware, primarily the mounts for the fiber collimator head assemblies. The mounts were attached to a water-

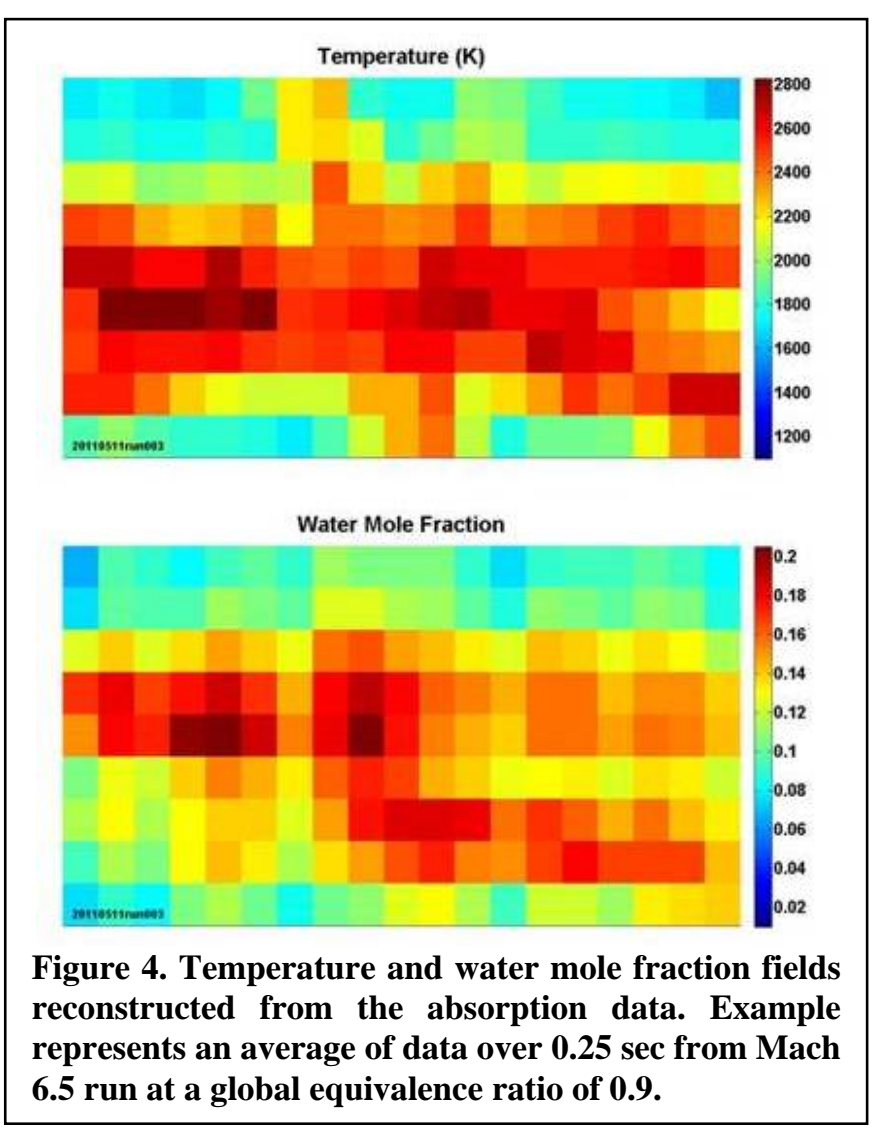
cooled substrate but not otherwise temperaturecompensated. Over the course of a run their differential heating lead to some beam walk. While beam walk was observed to greater and lesser degrees on essentially all runs it was not sufficient to prevent meaningful extraction of temperature and water concentration from the absorption signals.

High-frequency oscillations in the transmission signals accompanied the long term drift. These oscillations appear with peak-to-peak amplitudes roughly $10 \%$ of that of the mean and have spectral content up to the sampling frequency of $1 \mathrm{kHz}$. We note that the oscillations in the emission signal have similar frequency behavior. Taken together these observations suggest that the source of the oscillation is associated with the flow and not mechanical vibration. The transmission oscillations are most likely due to beam steering associated with small-scale density fluctuations along the lines of sight.

Figures 4 and 5 provide examples of tomographic reconstruction of the temperature and water mole fraction fields from the absorption data. The total area of each field reflects the total area of the opening at the exit plane of the combustor. The pixilation reflects the spatial grid employed in the reconstruction. Finer grid size simply encourages poor interpolation between the fixed LOS. At this point we make no attempt to smooth the images until we are satisfied with the maturity of the tomography algorithm. Figure 4 shows the temperature and corresponding water mole fraction for a $1 / 4$-second average during steady-state engine operation at a flight enthalpy corresponding to Mach 6.5 for a global equivalence ratio of 0.9 . Both images indicate - in a time-averaged sense - burning across the core of the flow with local hot spots. There is also evidence of port/starboard asymmetry. Figure 5 shows two sequential temperature fields from 1/4-second time averages spaced 20 milliseconds apart. Note the significant changes in spatial distribution. This reflects the intrinsic four-dimensional nature of the turbulent combustion flowfield in a model scramjet engine. Previous work executed

6

American Institute of Aeronautics and Astronautics 
at WPAFB and elsewhere using wall-based measurements and chemical emission imaging reveals that even at low frequency sampling, cavity flameholding in scramjet combustors is characterized by spatiotemporal transients in the local heat release.

\section{Conclusion}

A TDLAS system was coupled to the exit plane of the HDRC HIFiRE 2 ground test engine. Absorption, optical emission and non-resonant transmission data were successfully collected during Phase II testing of this flow path. A

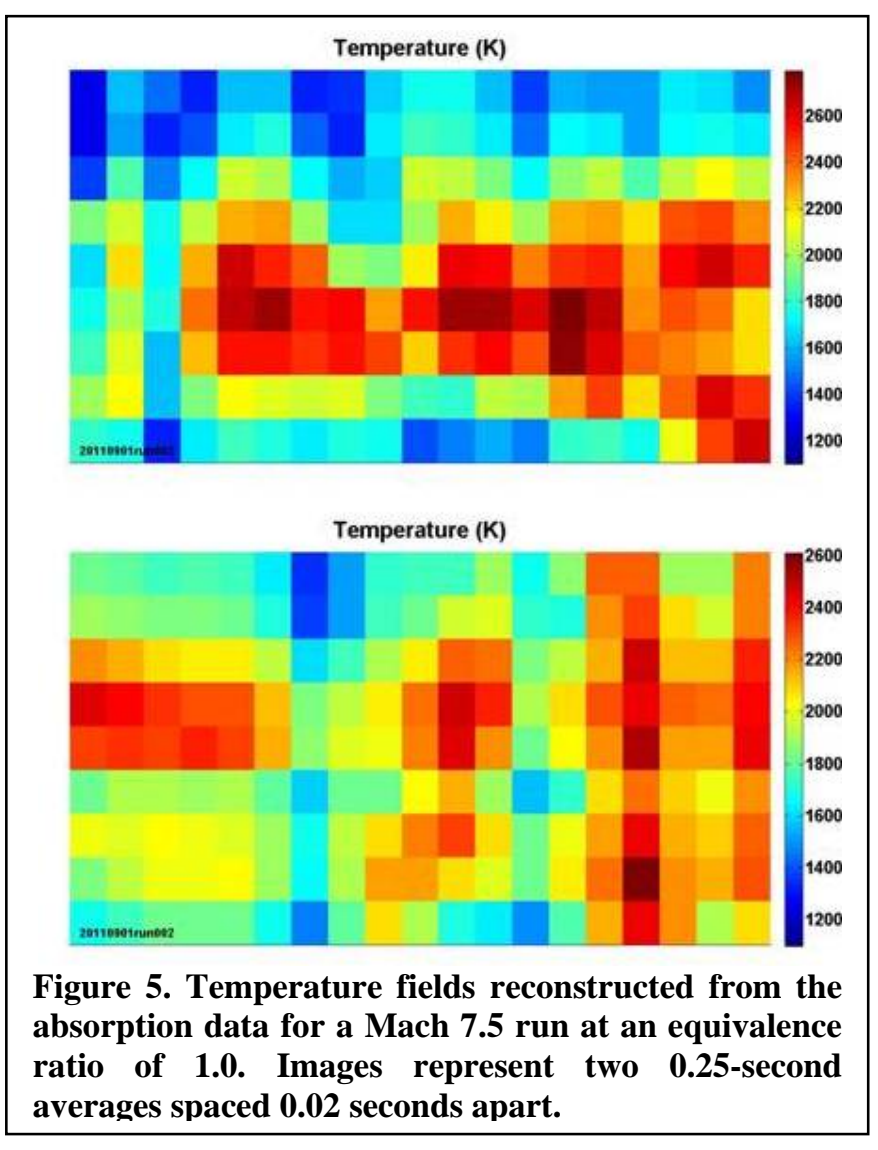
first pass at processing the data indicates that: 1) fine particulate effluent from the arc heater has negligible impact with regards to beam obscuration, 2) due to hardware thermal growth, some optical alignment drift was observed but is insufficient to prevent data analysis, 3) ambient water makes very little contribution to the absorption signal associated with the hypersonic flow, 4) radiative emission from the hot gases exiting the combustor tracks fueling of the engine and changes in wall temperature, and 5) tomographic reconstruction of a sample of data under "steady-state" engine conditions highlights the intrinsic 4-D character of supersonic turbulent combustion. Detailed analysis of the acquired data is an ongoing process leading to comparisons with CFD simulations of this flowpath, low-order modeling of the same, and previous work on engines of the small scale. We anticipate further refinements of the tomographic reduction algorithm as well. All of these efforts will be reported in future papers.

\section{Acknowledgments}

This work was supported in part by APTT (Tom Fetterhoff Executing Agent), and the Air Force Office of Scientific Research (Dr. John Schmisseur, Program Manager).

\section{References}

1 Jackson, K. R., Gruber, M., and Buccellato, S., "HIFiRE Flight 2 Overview and Status Update 2011,” AIAA Paper 2011$2202,2011$.

2 Sappey, A., Sutherland, L., Owenby, D., VanHoudt, P., Hannam, J., Zhao, Q., McCormick, P., Masterson, P., Estes, M., Williams, S., and Barhorst, T., "Flight-Ready TDLAS Combustion Sensor for Hypersonics," AIAA Paper 2009-7234, 2009.

3 Hass, N., Cabell, K., and Storch, A., "HIFiRE Direct-Connect Rig (HDCR) Phase I Ground Test Results from the NASA Langley Arc-Heated Test Facililty,” JANNAF Joint Subcommittee Meeting, Dec. 2009.

4 Cabell, K., Hass, N., Storch, A., and Gruber, M., "HIFiRE Direct-Connect Rig (HDCR) Phase I Scramjet Test Results from the NASA Langley Arc-Heated Scramjet Test Facility," AIAA Paper 2011-2248, 2011.

5 Storch, A. M., Bynum, M., Liu, J., and Gruber, M., "Combustor Operability and Performance Verification for HIFiRE Flight 2," AIAA Paper 2011-2249, 2011.

6 Williams, S., Barone, D., Barhorst, T., Jackson, K., Lin, K-C., Masterson, P., Zhao, Q., and Sappey, A. D., "Diode Laser Diagnostics for High Speed Flows,” AIAA Paper 2006-7999, 2006. 
7 Brown, M. S.,Barone, D., Barhorst, T. F., Eklund, D., Gruber, M., Mathur, T., and Milligan, R., "TDLAS-based

Measurements of Temperature, Pressure, and Velocity in the Isolator of an Axisymmetric Scramjet," AIAA Paper 2010-xxx, 2010.

8 Lindstrom, C., Tam, C-J., Givens, R., Davis, D., and Williams, S., "Diode Laser Absorption Tomography Using Data Compression Techniques," SPIE Vol. 6814, 2008, 68140W-1 - 68140W-17.

9 Lindstrom, C. D., Davis, D., Williams, S., Tam, C.-J., "Shock Train Structure Resolved with Absorption Spectroscopy Part 2: Analysis and CDF Comparison," AIAA J., Vol. 47, No. 10, 2009, 2379-2390.

10 It is easy to show that the radiation contribution from the combustor walls is negligible compared to the hot gases over the wavelength range captured by the photodetectors.

11 Sidky and Pan, Phys. Med. Biol. 53, 4777-4807 (2008).

12 Brown, M. S., Gruber, M., Kennedy, P., and Boyd, I., private communication, 2011. 